\title{
Thalamocortical Up States: Differential Effects of Intrinsic and Extrinsic Cortical Inputs on Persistent Activity
}

\author{
Pavlos Rigas and Manuel A. Castro-Alamancos \\ Department of Neurobiology and Anatomy, Drexel University College of Medicine, Philadelphia, Pennsylvania 19129
}

\begin{abstract}
During behavioral quiescence, the neocortex generates spontaneous slow oscillations that consist of Up and Down states. Up states are short epochs of persistent activity that resemble the activated neocortex during arousal and cognition. Although Up states are generated within the cortex, the impact of extrinsic (thalamocortical) and intrinsic (intracortical) inputs on the persistent activity is not known. Using thalamocortical slices, we found that the persistent cortical activity during spontaneous Up states effectively drives thalamocortical relay cells through corticothalamic connections. However, thalamic activity can also precede the onset of cortical Up states, which suggests a role of thalamic activity in triggering cortical Up states through thalamocortical connections. In support of this hypothesis, we found that cutting the connections between thalamus and cortex reduced the incidence of spontaneous Up states in the cortex. Consistent with a facilitating role of thalamic activity on Up states, electrical or chemical stimulation of the thalamus triggered cortical Up states very effectively and enhanced those occurring spontaneously. In contrast, stimulation of the cortex triggered Up states only at very low intensities but otherwise had a suppressive effect on Up states. Moreover, cortical stimulation suppressed the facilitating effect of thalamic stimulation on Up states. In conclusion, thalamocortical inputs facilitate and intracortical inputs suppress cortical Up states. Thus, extrinsic and intrinsic cortical inputs differentially regulate persistent activity, which may serve to adjust the processing state of thalamocortical networks during behavior.
\end{abstract}

Key words: slow oscillation; thalamocortical slice; thalamus; cortex; down state; arousal

\section{Introduction}

The thalamus and neocortex form recurrent networks (Sherman and Guillery, 1996; Steriade et al., 1997) that generate a variety of rhythmic electrical activities during different behavioral states (Steriade et al., 1993d; Castro-Alamancos and Connors, 1997). The different states of TC networks have profound consequences on signals flowing through them (Castro-Alamancos, 2004b).

During quiescent states, such as natural sleep and certain forms of anesthesia, cortical networks are not silent. Instead, they engage in spontaneous synchronized activity, such as "slow oscillations” (Metherate and Ashe, 1993; Steriade et al., 1993a). Slow oscillations are characterized by rhythmic cycles of synaptically mediated depolarization and action potentials (Up states), followed by decrease of synaptic inputs, leading to membrane hyperpolarization and cessation of firing (Down states). Up states correspond to recurrent synaptic network activity generated and maintained by balanced excitatory and inhibitory conductances (Shu et al., 2003). Up states are generated intrinsically in the neocortex (Steriade et al., 1993b; Sanchez-Vives and McCormick, 2000) and can recruit cortical targets, such as, for example, the thalamus (Steriade et al., 1993c) and the striatum (Cowan and

Received Jan. 1, 2007; revised Feb. 27, 2007; accepted March 16, 2007.

We thank Yoshie Tawara-Hirata for excellent technical assistance.

Correspondence should be addressed to Dr. Manuel A. Castro-Alamancos, Department of Neurobiology and Anatomy, Drexel University College of Medicine, 2900 Queen Lane, Philadelphia, PA 19129. E-mail: mcastro@drexelmed.edu.

DOI:10.1523/JNEUROSCI.0003-07.2007

Copyright $\odot 2007$ Society for Neuroscience $\quad$ 0270-6474/07/274261-12\$15.00/0
Wilson, 1994). The short epochs of persistent activity during cortical Up states resemble the activated state of neocortex during arousal and cognition (Moruzzi and Magoun, 1949; Steriade et al., 1993d; Castro-Alamancos, 2004b).

Slow oscillations consisting of Up and Down states occur spontaneously in isolated cortical slices of adult ferrets (SanchezVives and McCormick, 2000; Shu et al., 2003), rats (Cunningham et al., 2006), and mice (Castro-Alamancos and Rigas, 2002) bathed in a buffer that contains a concentration of divalent cations that resembles the "natural" CSF (i.e., $\sim 1 \mathrm{mM}\left[\mathrm{Ca}^{2+}\right]_{\mathrm{o}}$ and $\left.\left[\mathrm{Mg}^{2+}\right]_{\mathrm{o}}\right)$. Moreover, spontaneous recurrent network activity, consisting of Up and Down states, also arises in slices of developing mice [postnatal day 14 (P14) to P18] bathed in a "traditional" slice buffer (i.e., $\sim 2 \mathrm{mM}\left[\mathrm{Ca}^{2+}\right]_{\mathrm{o}}$ and $\left[\mathrm{Mg}^{2+}\right]_{\mathrm{o}}$ ) (Mao et al., 2001; MacLean et al., 2005).

Although Up states are generated intrinsically by cortical networks, an important question is how the cortical Up and Down states are affected by extrinsic synaptic thalamocortical activity and by intrinsic synaptic intracortical activity. Previous work has shown that Up states can be triggered or stopped by cortical stimulation (Shu et al., 2003). Moreover, a recent study found that thalamic stimulation at $>10 \mathrm{~Hz}$, in developing mouse slices bathed in traditional buffer, triggered cortical Up states that were identical to those occurring spontaneously (MacLean et al., 2005). Using thalamocortical slices from adult mice bathed in natural buffer, we further studied the impact of thalamic (extrinsic) and cortical (intrinsic) activity on Up states. We found that cortical and thalamocortical cells are actively engaged in sponta- 
neous cortical Up states and that intrinsic and extrinsic cortical inputs have distinct effects on Up states. Whereas extrinsic inputs trigger or enhance Up states, intrinsic inputs effectively suppress Up states.

\section{Materials and Methods}

Slices were prepared as previously described (Castro-Alamancos and Rigas, 2002; Castro-Alamancos et al., 2006) from adult ( $>7$ weeks) CD-1 or $\mathrm{BALB} / \mathrm{c}$ mice. Mice were deeply anesthetized with an overdose of ketamine hydrochloride ( $>100 \mathrm{mg} / \mathrm{kg}$ ) or sodium pentobarbital (>50 mg/ $\mathrm{kg}$ ). After losing all responsiveness to a strong tail pinch, the animal was decapitated, and the brain was rapidly extracted. Slices (400 $\mu \mathrm{m}$ thick) were cut in the thalamocortical plane (Agmon and Connors, 1991) using a vibratome. Slices were transferred to an interface chamber, in which they were bathed constantly $(1-1.5 \mathrm{ml} / \mathrm{min}$ ) with artificial CSF (ACSF) at $32.5^{\circ} \mathrm{C}$. The ACSF contained the following (in $\mathrm{mm}$ ): $126 \mathrm{NaCl}, 3.5 \mathrm{KCl}$, $1.25 \mathrm{NaH}_{2} \mathrm{PO}_{4}, 26 \mathrm{NaHCO}_{3}, 1 \mathrm{MgSO}_{4} 7 \mathrm{H}_{2} \mathrm{O}, 10$ dextrose, and $1 \mathrm{CaCl}_{2}$ $2 \mathrm{H}_{2} \mathrm{O}$. Single-unit and field potential recordings were made using highimpedance $(10-20 \mathrm{M} \Omega)$ and low-impedance $(\sim 0.5 \mathrm{M} \Omega)$ glass pipettes filled with ACSF, respectively. Blind whole-cell recordings were obtained from layer IV-III cells of somatosensory cortex (SI) using patch electrodes of 4-10 $\mathrm{M} \Omega$ impedance. The electrodes were filled with internal solution containing the following (in $\mathrm{mm}$ ): $135 \mathrm{~K}$-gluconate, $4 \mathrm{KCl}, 2$ $\mathrm{NaCl}$, 0.2 EGTA, 5 Tris-phosphocreatine, 0.3 Tris-GTP, 10 HEPES, and 4 MgATP (290 mOsm).

In some cases, the internal solution contained neurobiotin $(0.2 \%)$ to label the recorded cells. In other experiments, we used iontophoresis to inject neurobiotin in the thalamus to label its connections with the cortex. Iontophoresis was performed with a low-impedance glass pipette filled with neurobiotin ( $2 \%$ in $0.5 \mathrm{~m} \mathrm{~K}$-acetate) and consisted of current pulses $(1.5 \mu \mathrm{A})$ of $5 \mathrm{~s}$ duration delivered at $0.1 \mathrm{~Hz}$ during $15 \mathrm{~min}$. To allow transportation of the neurobiotin after injection, the slices were incubated in the recording chamber for $2-3 \mathrm{~h}$ after the iontophoresis before placing them in fixative. The slices were fixated in $4 \%$ paraformaldehyde with $1 \%$ glutaraldehyde and later cryoprotected with sucrose $(30 \%)$ and resectioned on a cryostat $(80 \mu \mathrm{m})$. Sections were incubated in $3 \%$ hydrogen peroxide, followed by $0.2 \%$ Triton X-100 and by incubation in $2 \%$ goat serum. Incubation with $\mathrm{ABC}$ reagent (Vector Laboratories, Burlingame, CA) occurs overnight. The following day, diaminobenzidine is applied to the sections. After color development, sections are mounted and cleared in xylene. Cells were later traced using Neurolucida software (Microbrightfield, Williston, VT). All procedures were reviewed and approved by the Animal Care Committee of Drexel University.

Concentric bipolar stimulating electrodes (FHC, Bowdoinham, ME) were used to electrically stimulate the thalamus and cortex. A glass micropipette pulled to a fine tip and filled with glutamate ( $100 \mathrm{~mm}$ in saline; $7.4 \mathrm{pH}$ ) was used to chemically stimulate the thalamus. Puffs of glutamate of different durations were ejected from the pipette by applying compressed air (30 psi) using a pressure valve (Harvard Apparatus, Holliston, MA) controlled by transistor-transistor logic pulses. The diameter of the pipette was adjusted so that it would not leak glutamate in the absence of a pressure pulse. To identify spontaneous Up states on-line and apply stimuli during the Up state, an analog threshold detector was used to detect $\mathrm{Up}$ states from the synchronous negative deflections of the field potential recording. To identify Up states off-line, a threshold was also used to detect $U p$ states from the synchronous negative deflections of the field potential recording. In every experiment, the accuracy of the detection was confirmed by visually inspecting the continuous recording off-line.

Data analyses were performed using Origin (OriginLab, Northampton, MA) and SPSS (SPSS, Chicago, IL) software. To measure spontaneous or evoked Up states from the field potential recordings, power spectrum analyses were derived by calculating fast Fourier transforms (FFTs), and the amplitude of the maximum negative peak of the field potential deflection was calculated. To measure Up states from the intracellular recordings, the action potentials were removed using a median filter, and the area of depolarization from the resting membrane potential, before the Up state, was calculated. The incidence of spontaneous Up states was
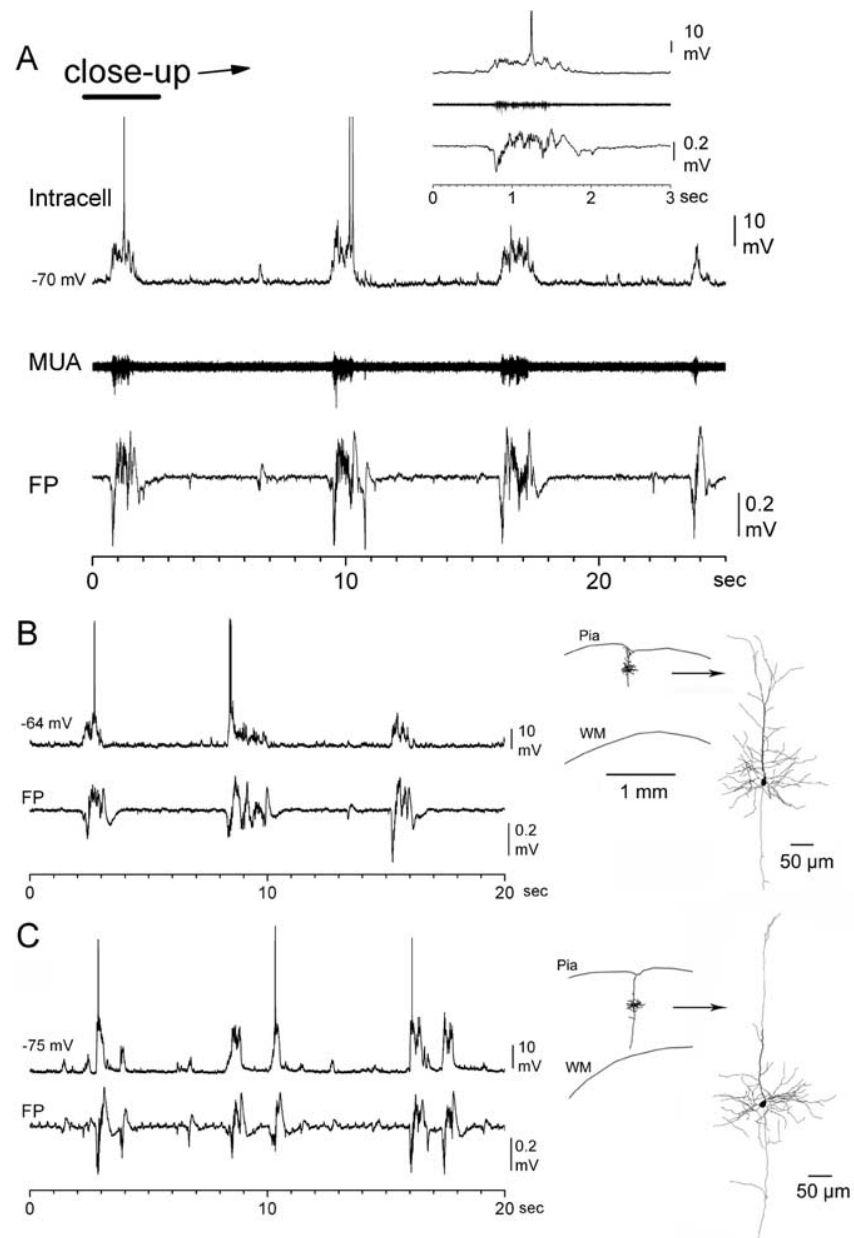

Figure 1. Spontaneous slow oscillations in SI cortex. A, Typical spontaneous Up and Down states observed with intracellular (whole-cell) and extracellular (population) recordings from layer IV-III in SI cortex of thalamocortical slices. The extracellular recording was low-pass (bottom trace) and high-pass (middle trace) filtered to reveal field potential (FP) and multiunit activity (MUA), respectively. $\boldsymbol{B}, \boldsymbol{C}$, Typical activity observed in two other slices in which the intracellular recorded cells were labeled and reconstructed. WM, White matter.

calculated using interevent time histograms (IETHs). Cross-correlations were calculated between the time stamps of thalamic spikes and the time stamps of the onset of cortical Up states. To calculate the shift predictor, the time stamps of one channel were shifted by $5 \mathrm{~s}$. For statistical analyses, data were first tested for normality based on the kurtosis and skewness of their distribution. If these values fell within the range of \pm 2 times their respective $\mathrm{SD}$, then the data were considered normally distributed, and parametric statistics were applied (ANOVA or $t$ test). Otherwise, we applied nonparametric statistics (Wilcoxon signed ranks for paired comparisons, Mann-Whitney for nonpaired comparisons, Kruskal-Wallis for multiple groups). Results are expressed as mean \pm SD unless otherwise indicated.

\section{Results}

\section{Spontaneous Up states in thalamocortical slices}

Using thalamocortical slices from adult mice, we conducted simultaneous intracellular (whole-cell) and extracellular (field potential and multiunit) recordings from microelectrodes placed in layers IV-III of the SI cortex. All of the cells reported in this study $(n=45)$ had overshooting action potentials, a resting membrane potential more negative than $-60 \mathrm{mV}$, and an average input resistance of $154 \pm 65 \mathrm{M} \Omega$ (measured with a $-0.1 \mathrm{nA}$ current pulse). Figure 1 shows typical spontaneous activity recorded from a sample of three cells taken from different experiments. In 
A

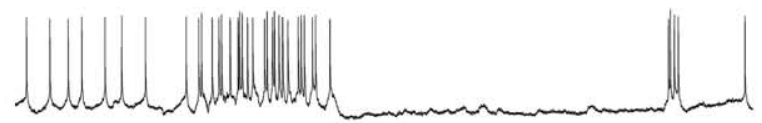

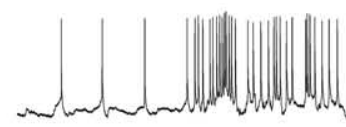

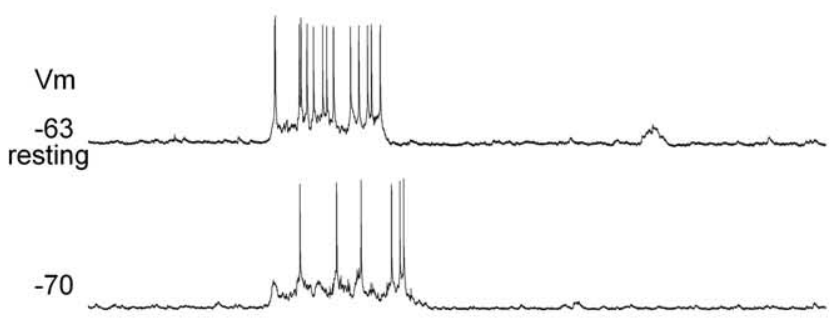

B

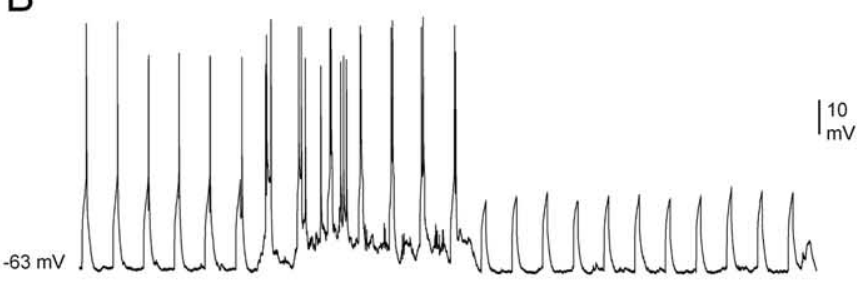

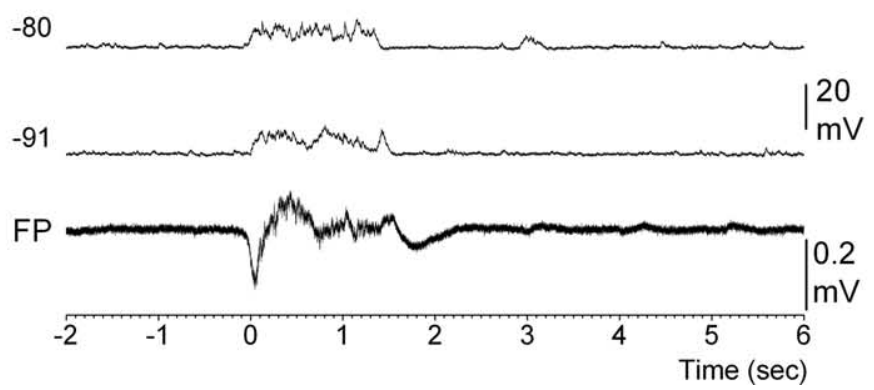

Figure 2. Spontaneous Up and Down states recorded at different membrane potentials. $\boldsymbol{A}$, Spontaneous Up states recorded from one cell at different membrane potentials produced by the injection of direct current. The top two traces correspond to the injection of positive current into the cell, whereas the bottom three intracellular traces correspond to the injection of negative current. Also shown at bottom is the average field potential (FP) recording corresponding to the six intracellular traces shown. $\boldsymbol{B}$, Example of the effect of short depolarizing current pulses ( 50 ms) before, during, and after the Up state. Note the absence of action potentials after the Up state. The simultaneously recorded FP corresponding to the intracellular trace is also shown in the bottom trace.

these slices, spontaneous Up and Down states recur at $\sim 0.1 \mathrm{~Hz}$. The Up state is readily detected in the field potential recording as a synchronous population (network) event that in the intracellular recording is characterized by barrages of postsynaptic potentials that depolarize the recorded neuron by $5-15 \mathrm{mV}$ for the duration of the network event, which on average lasts $1.3 \pm 0.5 \mathrm{~s}$ ( $n=12$ slices). During the Down state, cortical cells are relatively hyperpolarized, and there is little synaptic or network activity. These characteristics are indistinguishable from Up and Down states previously described in ferret slices (Sanchez-Vives and McCormick, 2000; Shu et al., 2003) and resemble those observed in vivo, although the Down state is usually longer and the Up state is shorter than in vivo (Steriade et al., 1993a). In some experiments, neurobiotin was included in the intracellular recording solution to label and subsequently trace the recorded cells $(n=5$ cells labeled) (Fig. $1 B, C$ ). The labeled cells were pyramidal-type cells located in layers IV-III.

To examine the characteristics of Up and Down states in individual cells, we moved the membrane potential to different levels with the intracellular injection of direct current (Fig. 2A). At depolarized potentials, the cells fired spontaneous action potentials immediately before and during an Up state but were silent for several seconds after the Up state (i.e., during the Down state). The reduced excitability during the Down state after each Up state was demonstrated by applying short depolarizing current pulses (Fig. 2 B). Before and during the Up state, the current pulse reliably evoked action potentials, whereas during the Down state, the same pulse was not capable of eliciting action potentials. Such a reduction in cell excitability immediately after the Up state may be related to a slowly recovering after-hyperpolarizing po- tential that is particularly obvious when the cell is depolarized by current injection (Fig. 2 A, top traces). This potential after the Up state reversed at quite hyperpolarized potentials (more negative than $-90 \mathrm{mV} ; n=3$ cells; data not shown), suggesting that it may be primarily mediated by a $\mathrm{K}^{+}$conductance.

\section{Up states in cortex are correlated with thalamic activity}

Simultaneous recordings from both cortex and thalamus in thalamocortical slices should reveal whether the thalamus is in any way engaged with the cortical Up states. Here we distinguish between TC slices and non-TC slices. TC slices are those that have intact thalamocortical and/or corticothalamic connections, so that electrical stimulation within the thalamus produces some effect in the cortex. Non-TC slices are those cut adjacent to the TC slices in the same experiment but do not contain intact thalamocortical or corticothalamic fibers, so that electrical stimulation in the thalamus produces no effect in the cortex. We first conducted labeling experiments to determine the presence of intact corticothalamic fibers in TC slices.

In a group of TC slices ( $n=8$ slices from eight mice), we confirmed the presence of intact corticothalamic connections between the SI cortex and ventrobasal (VB) thalamus by applying neurobiotin with iontophoresis in the VB thalamus. The iontophoresis pipette was placed in the area of the VB thalamus, in which electrical stimulation triggered field potential responses in layers IV-III of SI cortex. If the corticothalamic cells in SI cortex have intact fibers that reach the VB thalamus, this procedure should result in retrogradely labeled cells in layer VI of SI cortex. Indeed, in every TC slice tested $(n=8)$, neurobiotin applied in the VB thalamus of TC slices resulted in labeling of a band of 


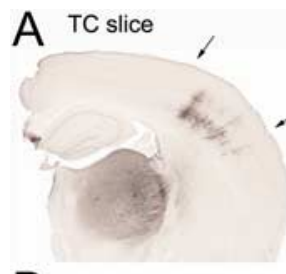

B
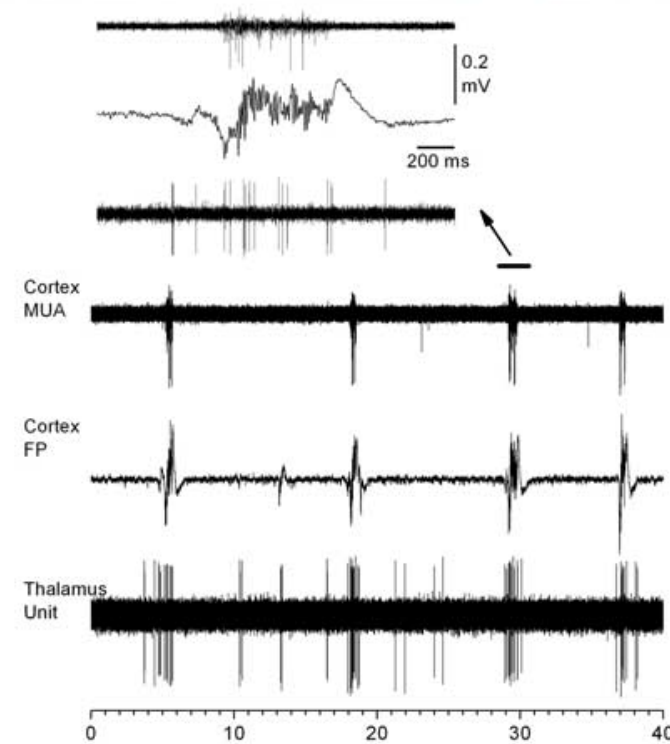

20

30
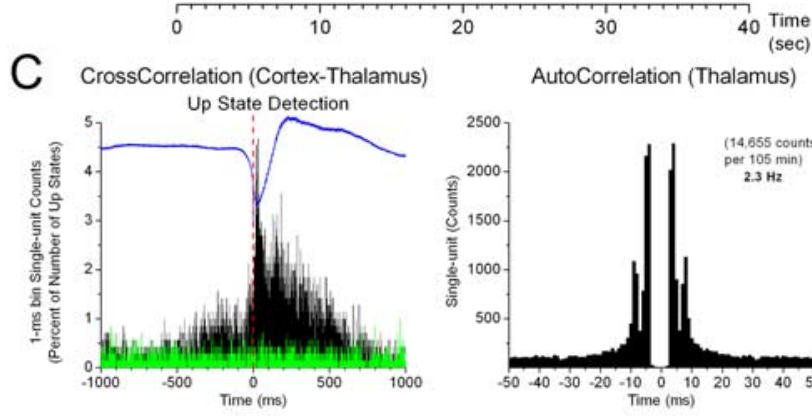

AutoCorrelation (Thalamus)

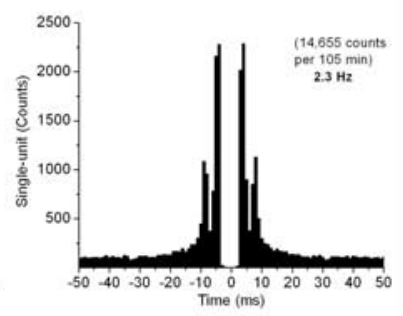

Figure 3. Spontaneous Up states in thalamocortical slices. $\boldsymbol{A}$, Effect of bulk iontophoresis injection of neurobiotin in the VB thalamus in three consecutive slices from the same animal. The left slice (TC slice) is the only one that showed labeling in the cortex. In particular, many corticothalamic cells were retrogradely labeled in layer VI of SI, indicating the presence of intact corticothalamic fibers between SI cortex and VB thalamus. The middle and right slices (non-TC slices) did not reveal any labeling in the cortex, indicating that connections between the thalamus and cortex had been severed in these slices. The three sections shown correspond to 80 $\mu \mathrm{m}$ cryostat sections taken from the middle of each of the three consecutive $400 \mu \mathrm{m}$ slices. The left slice is the most posterior of the three. $\boldsymbol{B}$, Simultaneous extracellular [field potential (FP) and multiunit activity (MUA)] recordings from layers IV-III of SI cortex and single-unit recording from the VB thalamus of a TC slice during spontaneous Up states. Note the firing of the VB thalamocortical cell in relation to the cortical Up states. $C$, Cross-correlation between the cortical Up state and the VB thalamocortical cell (left). The autocorrelation of the VB cell activity is also shown (right). The correlograms were calculated using continuous spontaneous activity obtained during $105 \mathrm{~min}$. This represents an average $2.3 \mathrm{~Hz}$ firing rate for the VB cell. The onset of the cortical Up state was taken as the reference marker (red dashed line) time stamp for the cross-correlation. The cross-correlation overlaid in green is shifted, representing spurious correlations. Overlaid (blue trace) on the cross-correlation is the average field potential of the detected Up states.

pyramidal cells in layer VI (i.e., corticothalamic cells). An example of this labeling is shown in Figure $3 \mathrm{~A}$ (left; the cortical area of retrograde labeling is marked by arrows).

In some experiments ( $n=8$ slices in four mice), we also applied neurobiotin in the VB thalamus of adjacent non-TC slices (i.e., those slices in which electrical stimulation of the VB thalamus produced no response in the SI cortex). In non-TC slices, the iontophoresis pipette was placed in the middle of the $\mathrm{VB}$ thalamus or in areas equivalent to the TC slices. We did not find any retrogradely labeled cells in the neocortex of non-TC slices despite extensive labeling in the thalamus. Two examples are shown in Figure $3 A$ (middle, right). Thus, the labeling example in Figure $3 A$ shows three consecutive slices from the same animal (the middle $80 \mu \mathrm{m}$ cryostat section of each of the three $400 \mu \mathrm{m}$ slices is shown). Note that only the TC slice (left), which was the only slice that produced field potential responses in cortex when stimulated in the thalamus, showed retrogradely labeled cells in the cortex. It is worth mentioning that our slicing procedures are quite standardized, and we could predict with confidence, before histological and/or electrophysiological confirmations, which slice in the order of slicing was the most effective TC slice. It is logical that we found intact corticothalamic connections in these slices because electrical stimulation of the SI cortex in TC slices produces synaptic responses in VB thalamocortical relay cells that are mediated by corticothalamic synapses (Kao and Coulter, 1997; Castro-Alamancos and Calcagnotto, 1999, 2001). These results demonstrate that TC slices contain intact (descending) corticothalamic connections, in addition to the well known intact (ascending) thalamocortical connections. Through descending corticothalamic connections, cortical Up states may drive thalamocortical relay cells, and through ascending thalamocortical connections, relay cells may affect cortical Up states.

Using TC slices, we set out to determine whether spontaneous Up states in SI cortex had any relationship with the activity of thalamocortical relay cells in VB thalamus. Field potential and multiunit activity was recorded through one electrode in SI cortex, and single units were recorded through another electrode in the VB thalamus. In TC slices, spontaneous cortical Up states were accompanied by activity in the VB thalamus (Fig. $3 B$ ), indicating that the cortical Up state was driving the activity of thalamocortical cells via corticothalamic connections. The VB thalamocortical cell shown in Figure 3 had an overall spontaneous firing rate of $2.3 \mathrm{~Hz}$ (measured over $105 \mathrm{~min}$ ) and, as denoted by the peaks of $<10 \mathrm{~ms}$ in the autocorrelogram (Fig. 3C, right), tended to fire frequently in bursts. To measure the relation between the spontaneous cortical activity and the thalamic activity, we computed cross-correlations between the cortical Up states and the thalamic spikes (Fig. 3C, left). Each cortical Up state was detected during its onset from the field potential recording. This time stamp was used as the marker to correlate with the thalamic spikes. The cross-correlation revealed large peaks that were well above a shifted correlogram (shift predictor) for the same activity (overlaid in green). In this experiment, the thalamic firing followed very faithfully the cortical Up state, indicating that the cortical Up state was driving synchronous thalamic activity. Moreover, the thalamic firing could begin $\sim 500 \mathrm{~ms}$ before the Up state was detected in the cortex. This suggests that thalamic activity may be triggering some of the cortical Up states, because it precedes them. An average of all of the detected field potential Up states for this experiment is also overlaid on the crosscorrelogram (blue trace). In other experiments with TC slices, the cortical Up states were not at all preceded by any thalamic activity. Instead, the thalamic activity followed very reliably the cortical Up state (Fig. $4 A$ ). In these cases, the cortex was driving the thalamocortical cells in the VB thalamus via corticothalamic connections, but there was no indication of thalamocortical cell firing preceding the cortical Up states.

In experiments with non-TC slices, there was no relation between the thalamic activity and the cortical Up states (Fig. $4 B$ ). In 

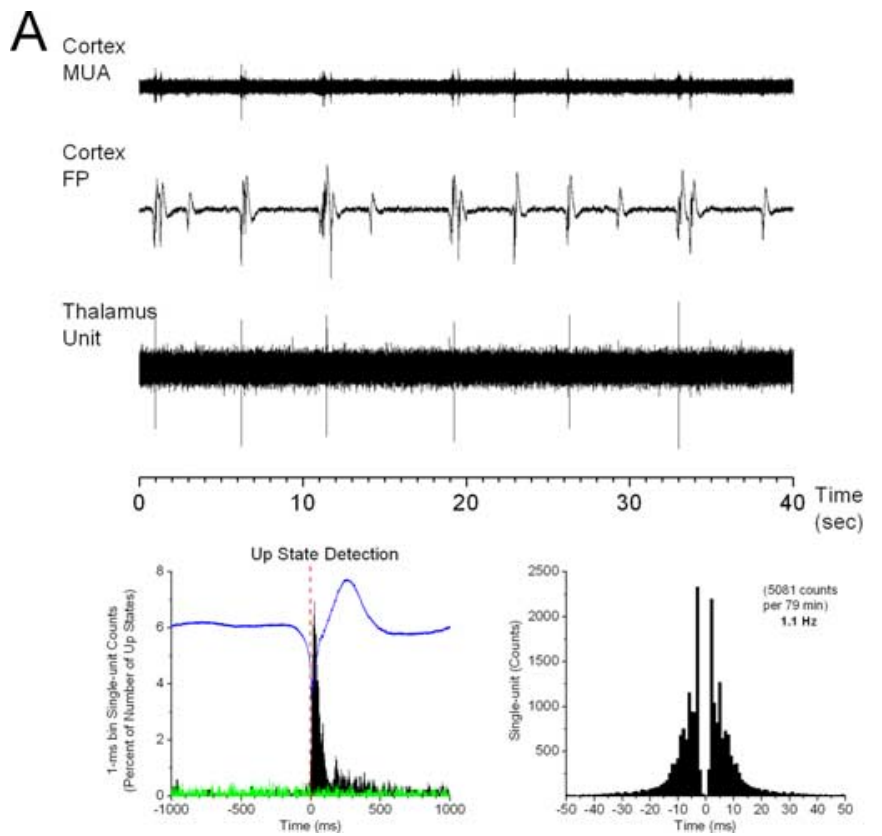
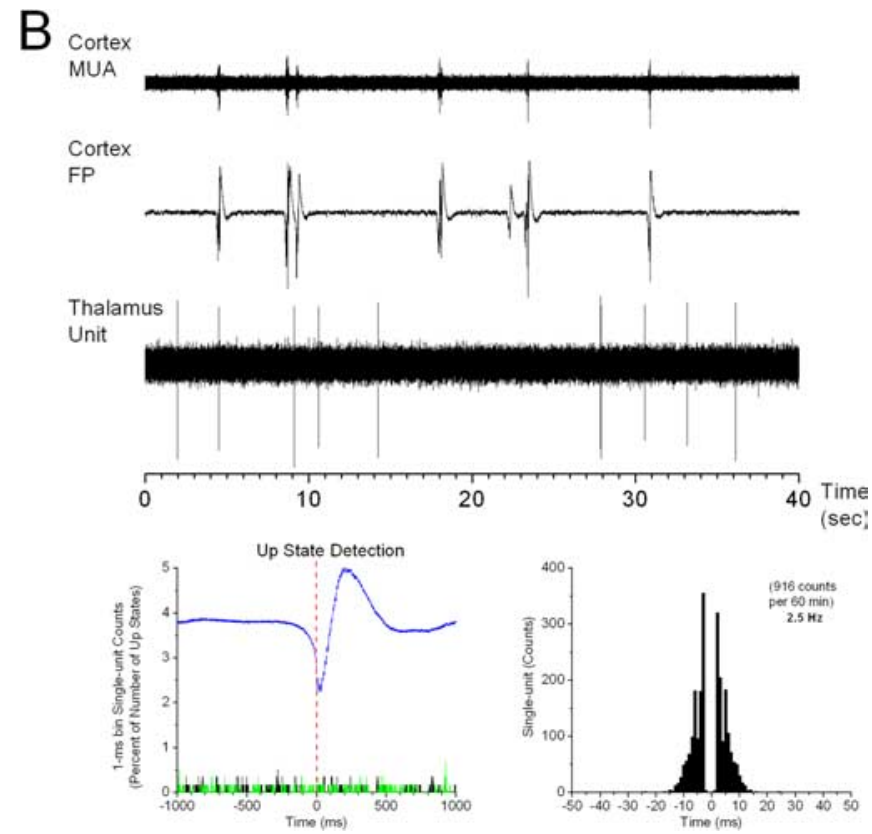

Figure 4. $\quad \boldsymbol{A}, \boldsymbol{B}$, Additional cross-correlation examples. Specific details for each panel are the same as for Figure 3 . The example in $\boldsymbol{A}$ corresponds to a TC slice, whereas the example in $\boldsymbol{B}$ corresponds to a non-TC slice.

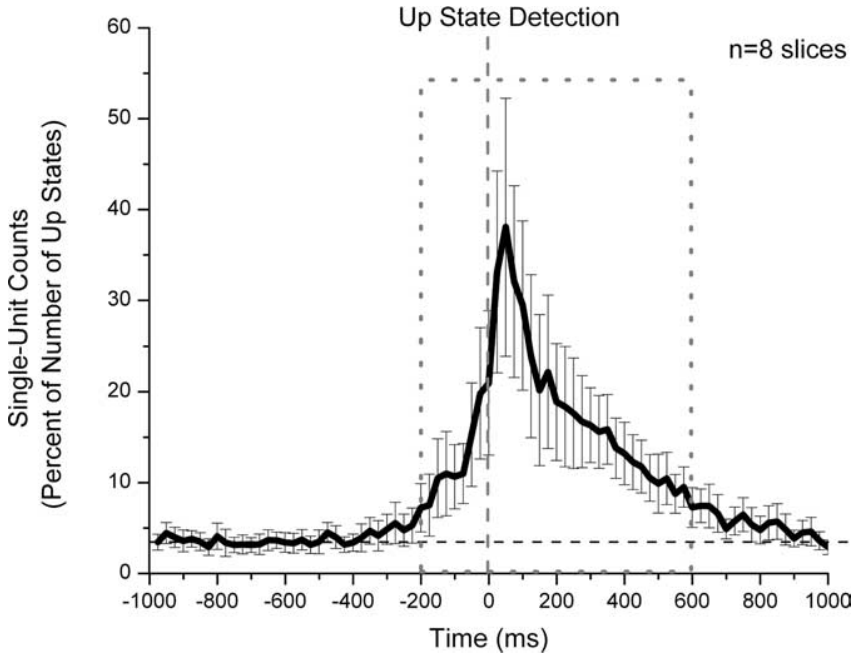

Figure 5. Population data showing the average (mean \pm SD) cross-correlation between cortical Up states and VB thalamocortical cells in TC slices that showed a significant crosscorrelation (above a shifted cross-correlation) between the cortical and thalamic activities ( $n=$ 8 slices). The dashed gray box represents the interval that revealed a significant crosscorrelation in different slices.

these cases, the activity in the SI cortex and VB thalamus occurred independently of each other. Figure 5 shows population data from TC slices that had a significant cross-correlation between the cortical Up state and the thalamocortical cells in VB thalamus ( 25 ms bin; mean $\pm \mathrm{SD} ; n=8$ ). A gray dashed box marks the time before and after the Up state detection that showed a significant $(p<0.05)$ increase in thalamic activity above the shifted correlogram.

These results demonstrate that cortical Up states effectively drive related activity in thalamocortical cells via corticothalamic connections. Moreover, in some cases the activity of thalamocortical cells in VB thalamus precedes the cortical Up states, which suggests that thalamic activity may be triggering some of the cortical Up states. This last hypothesis was further tested next.
The thalamus affects the incidence of spontaneous Up states in the cortex

If spontaneous thalamic activity in VB thalamus is impacting the incidence of Up states in the SI cortex, then interrupting the connections between the thalamus and cortex should affect the occurrence of Up states in SI cortex. To test this possibility, we cut the connections between the thalamus and cortex in TC slices using a fine blade attached to a micromanipulator. Fifteen minutes after the cut, we began measuring the effect of this manipulation on the incidence of Up states in the SI cortex. We simultaneously measured the incidence of Up states in the medial portion of cortex (non-SI cortex) from the same slice, which does not receive intact thalamocortical input in TC slices (Fig. 6A). Furthermore, we made the same cut in non-TC slices from the same animals, which served as a control of the potential effects of the cutting procedure. A cut of the thalamic radiation in TC slices significantly reduced the incidence of Up states in the SI cortex $(n=12$ slices; $p<0.01)$ (Fig. $6 B$ ) but not in the medial portion of the same slice, which was simultaneously recorded. Moreover, a cut of the thalamic radiation had no effect on spontaneous Up states in non-TC slices ( $n=12$ slices) (Fig. $6 B$ ). IETHs of the spontaneous Up states in TC slices revealed that the cut significantly reduced the number of cortical Up states occurring at intervals of $<12$ s $(n=12$ slices; $p<0.05)$ and significantly enhanced the number of Up states occurring at intervals of $>30 \mathrm{~s}(p<0.05)$ (Fig. 6C). None of these changes were observed in the SI cortex of non-TC slices ( $n=12$ slices) (Fig. $6 C$ ), indicating that the reduction of $\mathrm{Up}$ state incidence was caused by eliminating connections between thalamus and cortex and not by other nonspecific effects related to the cutting procedure. A power spectrum FFT analysis of the Up states in SI cortex of TC and non-TC slices showed no differences before and after the cut ( $n=12$ slices per group) (Fig. $6 D$ ). This indicates that although the cut of the thalamic radiation suppressed the incidence of Up states, those Up states were similar before and after the cut (Fig. 6D). 
Effects of thalamic and cortical stimulation on triggering Up states The previous results indicate that thalamic activity can precede or follow the onset of spontaneous Up states in SI cortex and that a cut that interrupts connections between thalamus and cortex reduces the incidence of spontaneous Up states in SI cortex. This suggests that thalamic activity may have a role in triggering cortical Up states. If this is the case, stimulating the thalamus should effectively trigger cortical Up states.

We compared the ability of thalamic and cortical electrical stimulation to trigger Up states in SI cortex. To avoid stimulating thalamocortical fibers within the neocortex, the cortical stimulating electrode was placed in the top layers, between layers II and III, and $0.5-1 \mathrm{~mm}$ away from the field potential and whole-cell recording electrodes. Thus, two different stimulating electrodes were placed in the slice, one in the VB thalamus and another in the top layers of SI cortex. Input/output (I/O) curves were derived by varying the intensity of the stimulation applied (10-150 $\mu \mathrm{A})$. An example of such an experiment for a fast-spiking cell (FS cell; putative interneuron) is shown in Figure 7. Because Up states are population events, the effects of electrical stimulation on Up states were similar in regular-spiking and fast-spiking cells. Electrical stimulation of the cortex or thalamus evoked a typical short-latency EPSP that increased with intensity, and this could be followed by a longer-latency Up state. Interestingly, only low-intensity $(10-20 \mu \mathrm{A})$ stimulation of the cortex triggered an Up state, whereas increasing the cortex stimulation intensity to $>20 \mu \mathrm{A}$ resulted in a stronger short-latency EPSP that triggered an action potential and was followed by a robust IPSP, but an Up state was not produced. In contrast, increasing the thalamus stimulation intensity simply increased the likelihood of triggering an Up state (Fig. 7A).

Figure $7 B$ shows the average intracellular responses evoked by stimulating the cortex, the thalamus, or both together (cortex + thalamus) at different intensities for the FS cell in Figure $7 A$ (15 trials are averaged, and action potentials were removed using a median filter). As quantified later, results in regular-spiking cells were similar. The average depolarization evoked by each stimulation is compared with the average depolarization produced by the same number of spontaneous Up states. Thus, in this experiment, stimulation of the cortex triggered an Up state at 15 $\mu \mathrm{A}$ but not at higher intensities (Fig. $7 B$, red trace). The triggered Up states were identical to those occurring spontaneously (i.e., an average of 15 spontaneous Up states is overlaid for comparison) (Fig. $7 B$, light gray trace). In contrast, stimulation of the thalamus began triggering Up states at intensi-
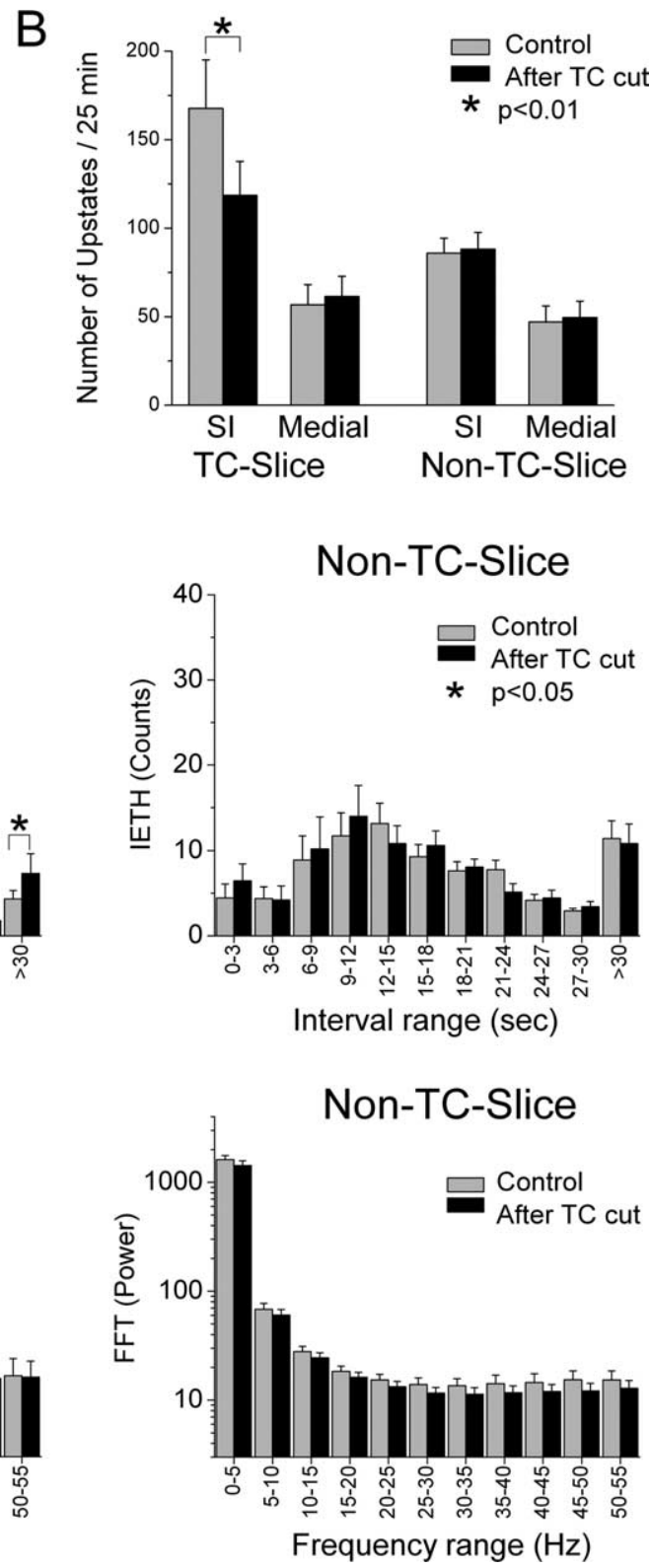

Figure 6. Effects of disconnecting the thalamus from the cortex on cortical Up states. $\boldsymbol{A}$, Photomicrograph of a TC slice preparation taken in the recording chamber showing the simultaneous recording sites in SI cortex (SI) and non-SI cortex (Medial) and the location at which a cut was made in the thalamic radiation to interrupt the connections between thalamus and cortex. $\boldsymbol{B}$, Effect of a thalamic radiation cut on the incidence of Up states in the SI cortex and in the simultaneously recorded medial non-SI cortex of both TC and non-TC slices. C, IETHs of the spontaneous Up states in SI cortex of TC and non-TC slices before and after a cut of the thalamic radiation. D, Power spectrum FFT analysis of the spontaneous Up states in SI cortex of TC and non-TC slices before and after a cut of the thalamic radiation. Error bars represent SD.

ties as low as $10 \mu \mathrm{A}$, albeit with low probability. Hence, the average depolarization was lower than that of spontaneous Up states. As the intensity of the thalamic stimulation increased to $>20 \mu \mathrm{A}$, the average depolarization evoked was similar to that of the spontaneous Up states, indicating that an Up state had been triggered in each stimulus trial. Simultaneous stimulation of the thalamus and cortex at low intensities resulted in a stronger depolarization than stimulating the thalamus or cortex separately, indicating a summation of their effects. However, simultaneous stimulation of the thalamus and cortex at higher intensities resulted in a reduced depolarization compared with the thalamus stimulation alone, indicating that the cortex stimulation was suppressing the ability of the thalamus 
A Cortex Stim
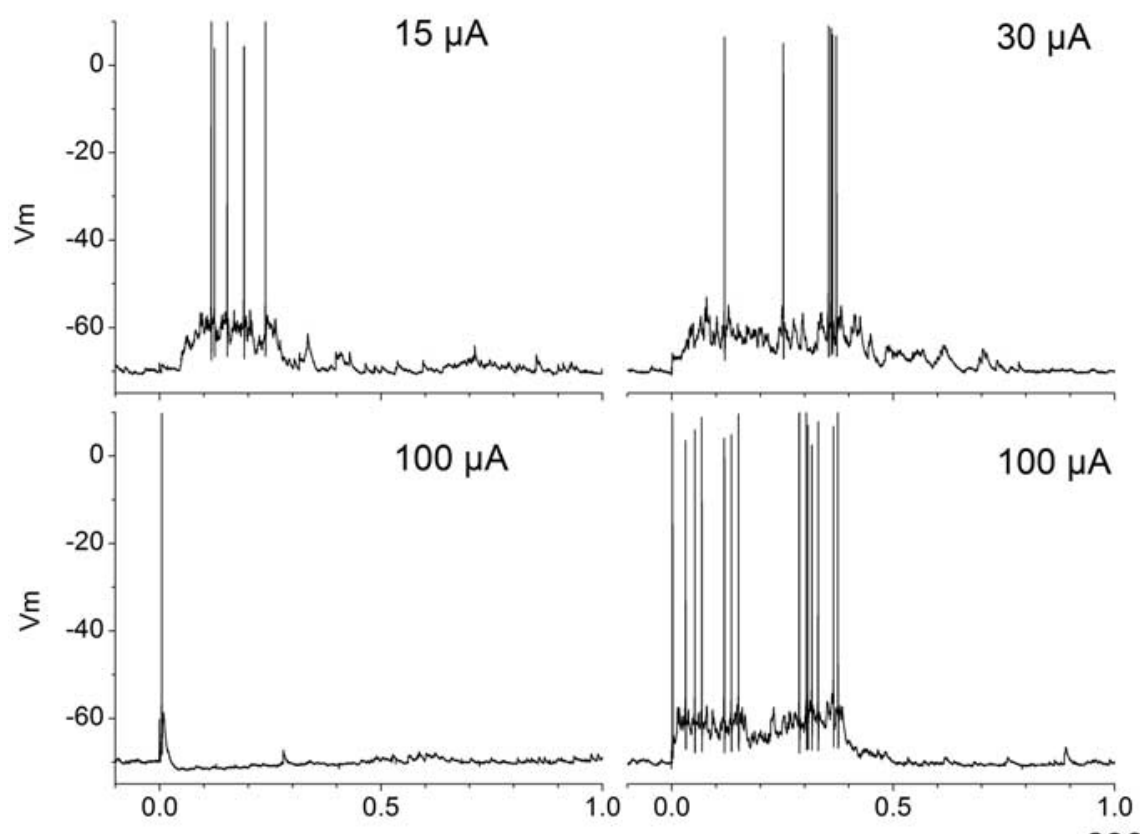

B

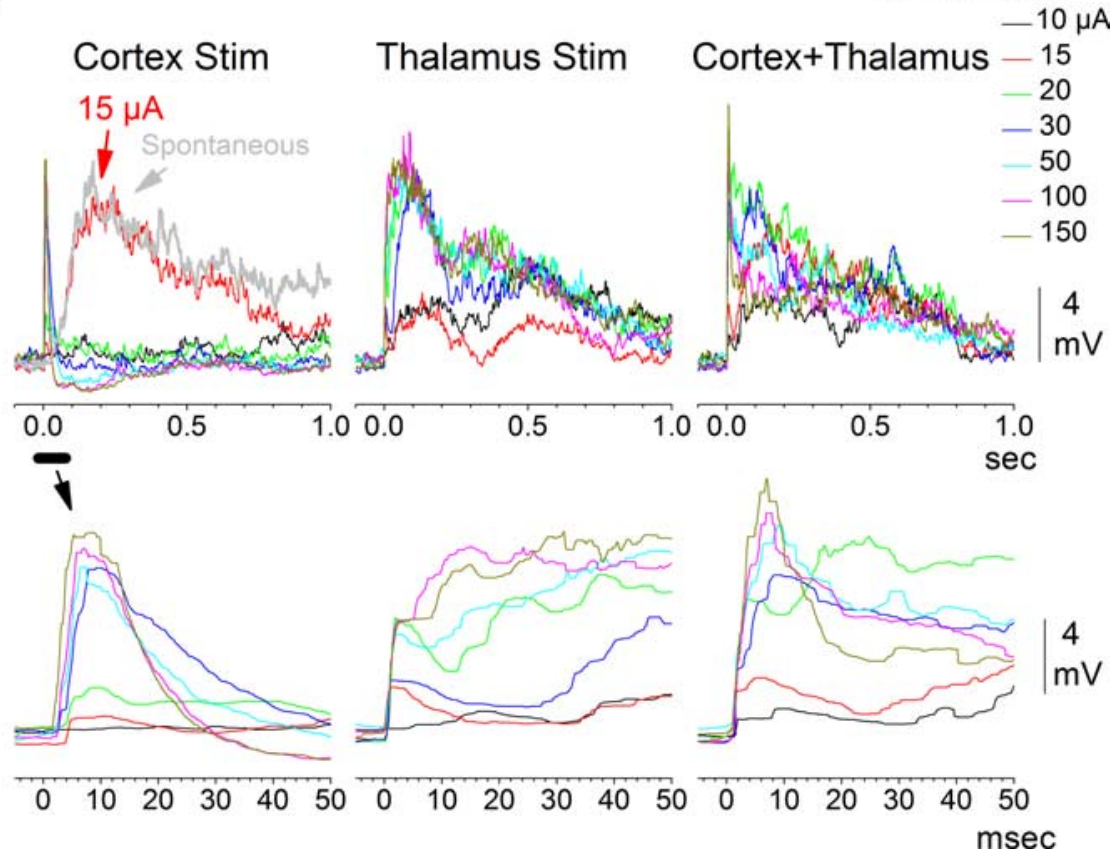

Figure 7. Effect of thalamus and/or cortex electrical stimulation (Stim) at different intensities on triggering cortical Up states. $\boldsymbol{A}$, Examples of single-trial responses of an FS cell to thalamus and cortex stimulation at different intensities. $\boldsymbol{B}$, Average of intracellular responses to thalamus, cortex, or cortex + thalamus stimulation at different intensities. Each trace is the average of 15 trials, and the action potentials were removed with a median filter. Overlaid in the left panel is the average of 15 spontaneous Up states for comparison (light gray trace). Bottom, Close-up of the top showing the short-latency responses.

slices) (Fig. $8 \mathrm{~A}$ ). This measure is simple to obtain, because Up states are easily identifiable events. To be counted as an Up state, the evoked response should meet two criteria. It should be phase locked to the stimulus (i.e., occur at a fairly constant latency on each trial) and have amplitude and duration similar to spontaneous Up states in the same experiment. As shown in Figure $8 A$, the probability of triggering an Up state from the cortex became nil as the stimulation intensity increased. Thus, there was a significant difference between lower- $(10-20 \mu \mathrm{A})$ and higher $(50-150$ $\mu \mathrm{A})$-intensity stimulation of the cortex in the probability of triggering an Up state ( $p<0.01 ; n=12$ slices). In contrast, the probability of evoking an Up state from the thalamus increased as function of intensity $(p<0.01$; lower vs higher intensity; $n=12$ slices). For the cortex + thalamus stimulation, the probability of triggering an Up state at low intensities $(10-30 \mu \mathrm{A})$ was approximately the sum of the cortex and thalamus stimulations delivered separately, but as the intensity increased, the probability was less than the thalamus stimulation alone. Thus, at intensities of $>50 \mu \mathrm{A}$, the cortex stimulation significantly suppressed the ability of the thalamus to trigger Up states $(p<$ 0.01 ; cortex + thalamus vs thalamus for $100-150 \mu \mathrm{A} ; n=12$ slices).

The second measure we obtained consisted of calculating the area of depolarization above the baseline membrane potential (prestimulus $V_{\mathrm{m}}$ ) for the intracellular recordings ( $n=7$ cells) (Fig. $8 B$ ). The area of depolarization evoked by each stimulus was compared with the area of depolarization of spontaneous Up states (Fig. $8 B$, blue). This comparison produced results similar to the probability measure described above. Thus, for the cortex stimulation, at low intensities, the area of depolarization was equivalent to that produced by spontaneous Up states [not significant (n.s.); cortex vs spontaneous Up states for $10-20 \mu \mathrm{A}]$, but as the intensity increased, the area of depolarization was strongly suppressed ( $p<0.01$; cortex vs spontaneous Up states for $30-150 \mu \mathrm{A}$ ). For the thalamus stimulation, the area of depolarization increased as a function of intensity and rapidly reached a value

to trigger Up states. Population data from multiple experiments are described below and shown in Figure 8.

To compare the efficacy of thalamic and cortical electrical stimulation at triggering Up states as a function of intensity, we obtained several measures from intracellular and field potential recordings. The first measure was the probability of triggering an Up state based on $15-30$ trials per stimulus intensity $(n=12$ equivalent to spontaneous Up states. For the simultaneous stimulation of the cortex + thalamus, at low intensities, the area of depolarization was equivalent to that produced by spontaneous Up states (n.s.; cortex + thalamus vs spontaneous Up states for $10-30 \mu \mathrm{A}$ ), but as the intensity increased, the area of depolarization was significantly less than that of spontaneous Up states $(p<0.05$; cortex + thalamus vs spontane- 


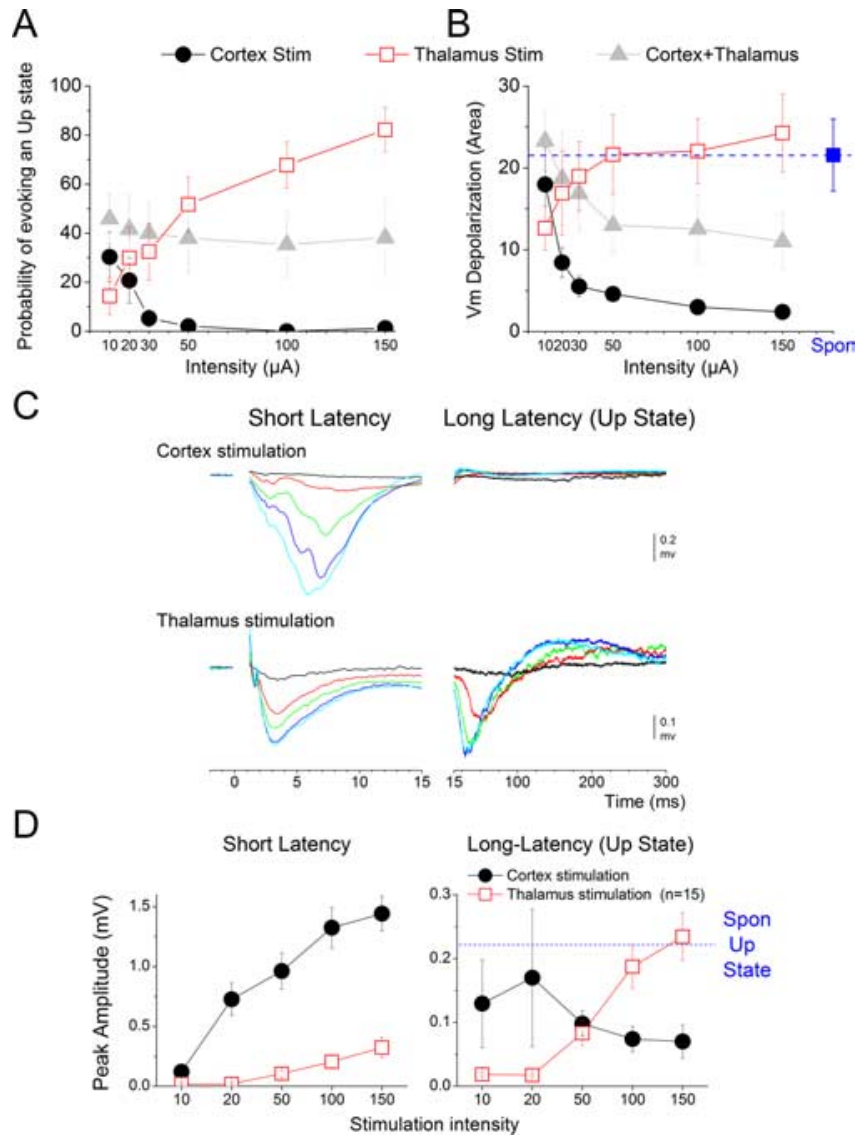

Figure 8. Population data of $\mathrm{I} / 0$ curves of thalamus and cortex stimulation (Stim) on Up states. $\boldsymbol{A}$, Probability of triggering a cortical Up state in response to thalamus stimulation, cortex stimulation, or both together as a function of the stimulation intensity. $\boldsymbol{B}$, Area of depolarization above the baseline membrane potential (prestimulus $V_{m}$ ) for intracellular recordings as a function of the stimulation intensity in thalamus, cortex or both together. Also shown for comparison is the area of depolarization produced by spontaneous Up states in the same experiments (Spon; blue symbol). C, Example of field potential traces evoked by thalamus or cortex stimulation at different intensities. Each trace is the average of 20 trials at 10,20,50, 100, and $150 \mu \mathrm{A}$. The left shows the short-latency response and the right shows the long-latency response, which reflects the Up state. $\boldsymbol{D}$, Peak amplitude of short- and long-latency responses evoked by thalamus or cortex stimulation at different intensities. The average amplitude of spontaneous Up states is shown as a blue dashed line in the right panel for comparison with the evoked longlatency responses. Error bars represent SD.

ous Up states for $50-150 \mu \mathrm{A}$ ). This indicates that the cortex stimulation was suppressing the ability of the thalamus to trigger Up states.

The third measure we obtained from an additional group of experiments ( $n=15$ slices) (Fig. $8 C, D)$ consisted of measuring the peak amplitude of short- (2-10 ms) and long (15-500 ms)latency field potential responses (Fig. $8 C, D)(n=15$ slices). The peak amplitude of the long-latency field potential response provides a simple way to measure Up states by comparing this amplitude with the amplitude of spontaneously occurring Up states (Fig. 8C,D). Indeed, the results obtained with this measure were similar to those obtained by measuring the probability of triggering an Up state (Fig. $8 A$ ) and the area of depolarization (Fig. $8 B$ ). The short-latency response increased as a function of stimulation intensity and was larger for the cortex than for the thalamus stimulation. In contrast, the long-latency response, representing the Up state, showed different effects for the cortex and thalamus stimulation. For the cortex stimulation, at low intensities (10-20 $\mu \mathrm{A})$, the long-latency response was variable but larger than at higher intensities $(50-150 \mu \mathrm{A})$. Thus, the long-latency response evoked by cortex stimulation was suppressed as the intensity increased. In contrast, for the thalamus stimulation, the amplitude of the long-latency response simply increased as a function of intensity.

In conclusion, these results indicate that the likelihood of triggering an Up state from the thalamus increases rapidly with intensity, whereas the likelihood of triggering an Up state from the cortex is high at low intensities and rapidly decreases to nil as the intensity increases. Moreover, simultaneous stimulation of thalamus and cortex revealed a summation effect at low intensities on triggering Up states and an inhibitory effect of moderate-tostrong cortex stimulation on the ability of the thalamus to trigger Up states.

\section{Thalamic activity triggers cortical Up states}

The excellent capability of thalamic electrical stimulation to evoke cortical Up states is in good agreement with the results showing that elimination of the thalamus reduces the incidence of Up states in the cortex and that Up states are correlated with thalamic activity. However, electrical stimulation of the thalamus will not only stimulate thalamocortical fibers ascending to the cortex but will also antidromically stimulate layer VI corticothalamic cells whose axons reach the thalamus. Thus, the intracortical synaptic collaterals of corticothalamic cells could be triggering the cortical Up states in response to thalamic electrical stimulation. To directly address this confound, we used puffs of glutamate delivered to the thalamus as a means of evoking cortical Up states. In TC slices, we first found a location at which electrical stimulation of the VB thalamus evoked an Up state in the cortex. This was followed by placing a glass pipette filled with glutamate in that location and then applying pressure pulses of increasing duration until a cortical Up state was reliably evoked or a maximum of $250 \mathrm{~ms}$ was reached. To assure that the glutamate was not spreading to the cortex, after finding an effective glutamate stimulation site in the VB thalamus, we moved the glutamate pipette to a location closer to the cortex (e.g., the thalamic radiation) and checked whether the glutamate pulse was still effective. In all cases, there was a localized region within the VB thalamus that would effectively evoke cortical Up states with glutamate puffs. Moving the glutamate pipette just a few hundred micrometers completely abolished its ability to evoke Up states.

Figure 9 shows two examples of cells that were subjected to these procedures. The left panels overlay thalamic stimulation trials produced by single electrical pulses, the middle panels overlay responses evoked by puffs of glutamate in the same thalamic site, and the right panels overlay spontaneously occurring Up states. Glutamate puffs were very effective at triggering Up states from the thalamus. The Up states triggered by thalamic glutamate had a relatively long but constant latency (within each experiment) from the onset of the glutamate pulse; the shortest latency that we obtained was $87 \mathrm{~ms}(221 \pm 80 \mathrm{~ms} ; n=6$ experiments $)$. The long latency surely reflects the mechanical nature of the pressure-dispensed glutamate. Once an effective thalamic site was found, the Up states evoked by glutamate occurred very reliably ( $>90 \%$ of trials) trial to trial and at a constant latency (Fig. 9). In some cases ( $n=4$ of 6 experiments), the Up state evoked by glutamate was clearly longer lasting than either the spontaneous or electrically evoked Up states (Fig. 9A). This likely reflects the sustained firing in thalamocortical cells produced by the long glutamate puff (data not shown), which contrasts with the synchronous and short jolt produced by the single electrical stimulus $(200 \mu \mathrm{s})$. These results demonstrate that thalamic activity (un- 

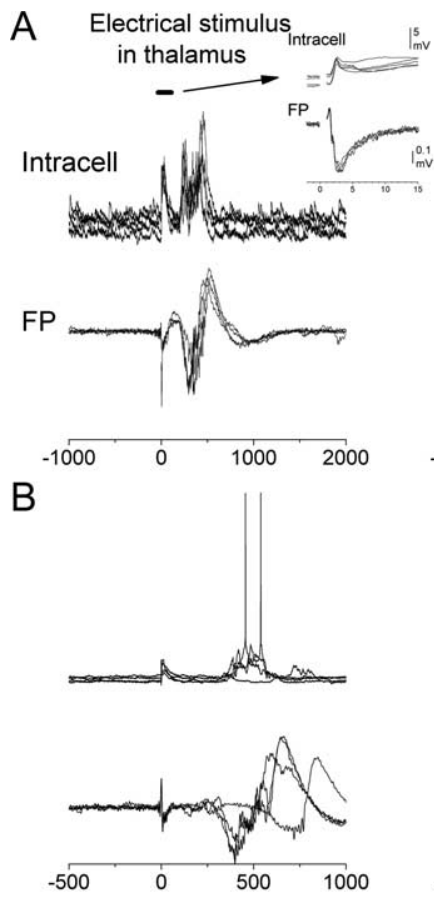
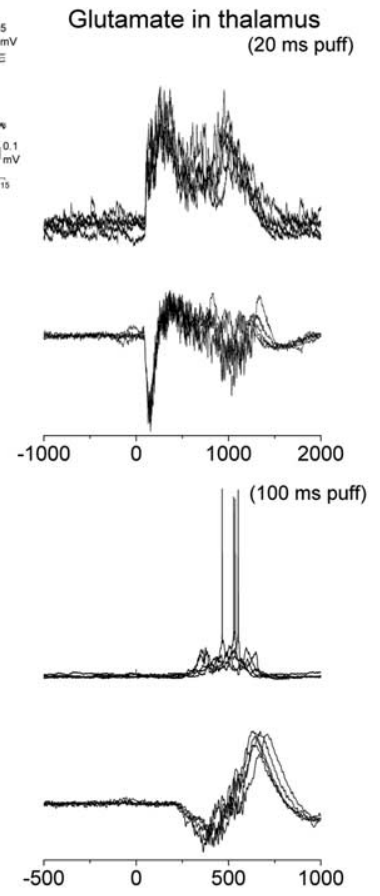

$-500$

Figure 9. Cortical Up states triggered by chemical stimulation of the thalamus. $\boldsymbol{A}$, Simultaneous intracellular and field potential (FP) recordings from layer IV-III of SI cortex. The panels overlay individual trial responses evoked by electrical stimulation of the thalamus (left), glutamate puffs in the thalamus (middle), or spontaneous Up states (right). Five trials are overlaid per panel. The stimulus onset is at zero. The glutamate puffs were $20 \mathrm{~ms}$ in duration. The left panel also shows a close-up of the short-latency response evoked by electrical stimulation of the thalamus (inset). $\boldsymbol{B}$, Same as in $\boldsymbol{A}$ for a different experiment. The glutamate puffs were $100 \mathrm{~ms}$ in duration.

contaminated by antidromic activation of corticothalamic cells) triggers cortical Up states. The results also suggest that sustained thalamic activity may have an added effect of prolonging the cortical Up states. In essence, thalamic activity has a net facilitating effect on cortical Up states.

\section{Effects of thalamic and cortical stimulation during spontaneous Up states}

The previous results indicate that thalamic activity has an overall facilitating effect on Up states, whereas cortical activity has a suppressive effect. To further test these hypotheses, electrical stimulation was delivered in the thalamus or in the cortex during the occurrence of spontaneous Up states. This should demonstrate whether thalamic stimulation enhances spontaneously occurring Up states and cortical stimulation suppresses them. To deliver the stimuli during the Up states, an analog threshold detector was used to detect the Up states on-line from the field potential recording. Also, once an Up state was detected, a lockout period assured that nothing was detected for $5 \mathrm{~s}$. Thus, stimuli were tested on the Up state at a rate of $<0.2 \mathrm{~Hz}$. In these experiments $(n=5)$, moderate- to high-intensity cortex and thalamus stimulation $(50-100 \mu \mathrm{A})$ was used. We compared the area of depolarization from intracellular recordings of spontaneous $U p$ states unaffected by any stimulus and spontaneous Up states affected by either a cortical or a thalamic stimulus delivered during the Up state. A total of 30-50 trials were averaged per cell and condition. The results revealed that spontaneous Up states were enhanced by thalamic stimuli and were suppressed by cortical stimuli (Fig. 10). This effect was observed in every cell tested ( $n=$ 5 cells), so that the area of membrane depolarization was $32 \%$ larger for spontaneous Up states affected by thalamic stimulation
Spontaneous
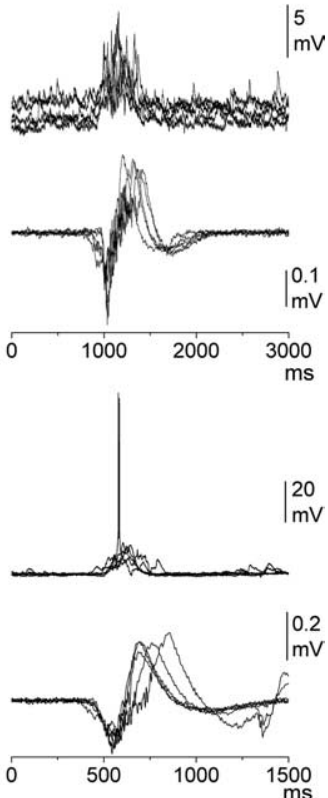

$\mathrm{ms}$

than for unstimulated spontaneous Up states $(p<0.05)$. Moreover, the area of membrane depolarization was $38 \%$ smaller for spontaneous Up states affected by cortical stimulation than for unstimulated spontaneous Up states $(p<0.05)$. In conclusion, thalamic stimulation has a facilitating effect on spontaneous Up states, whereas cortical stimulation has a suppressing effect on spontaneous UP states.

\section{Discussion}

Using thalamocortical slices of adult mice, we found that spontaneous Up states in SI cortex occur synchronously with firing in VB thalamocortical cells. Crosscorrelation analysis shows that most of the thalamic activity is driven by the cortical Up states. However, in some cases, the thalamic activity precedes the onset of cortical Up states, suggesting that it may be triggering them. In support of this possibility, elimination of the thalamus reduced the incidence of Up states in SI cortex. Moreover, electrical stimulation of the thalamus was very effective at triggering cortical Up states (much more so than cortical stimulation, which could only trigger Up states at very low intensities). In fact, when the thalamus and cortex stimulation were applied simultaneously, the cortex suppressed the ability of the thalamus to trigger Up states. The high effectiveness of thalamic activity at triggering cortical Up states was confirmed using glutamate puffs in the thalamus, which avoids the confounding effects of thalamic electrical stimulation (i.e., the antidromic activation of corticothalamic cells). Finally, stimulation during spontaneous Up states demonstrated the enhancing and suppressing effects of thalamic and cortical stimulation on cortical Up states, respectively.

\section{Spontaneous Up states in thalamocortical slices}

The results show that Up states generated within the cortex are synchronized with thalamic activity. Most of the correlated thalamic activity occurs after the onset of the cortical Up state, indicating that corticothalamic cells in SI cortex are driving thalamocortical cells in VB thalamus during the cortical Up state. The existence of a massive corticothalamic pathway from cortex to the VB thalamus is well documented [for reviews, see Sherman and Guillery (1996) and Castro-Alamancos (2004b)], and cortical afferents are the most abundant input to the thalamus (Guillery, 1971). In fact, it is estimated that for every thalamocortical axon traversing to the cortex, there are $\sim 10$ returning to the thalamus (Sherman and Guillery, 1996). We confirmed the existence of this massive pathway in our slices by applying neurobiotin in the VB thalamus. In TC slices, large numbers of layer VI corticothalamic cells were labeled retrogradely. Therefore, it is logical that the synchronous discharge of cortical cells during Up states drives thalamocortical cells that receive corticothalamic input and other cortical targets, as found in vivo (Steriade et al., 1993c; Cowan and Wilson, 1994). Interestingly, previous work using thalamocortical slices of developing mice (P14-P18) found no evidence of corticothalamic synchrony during spontaneous 


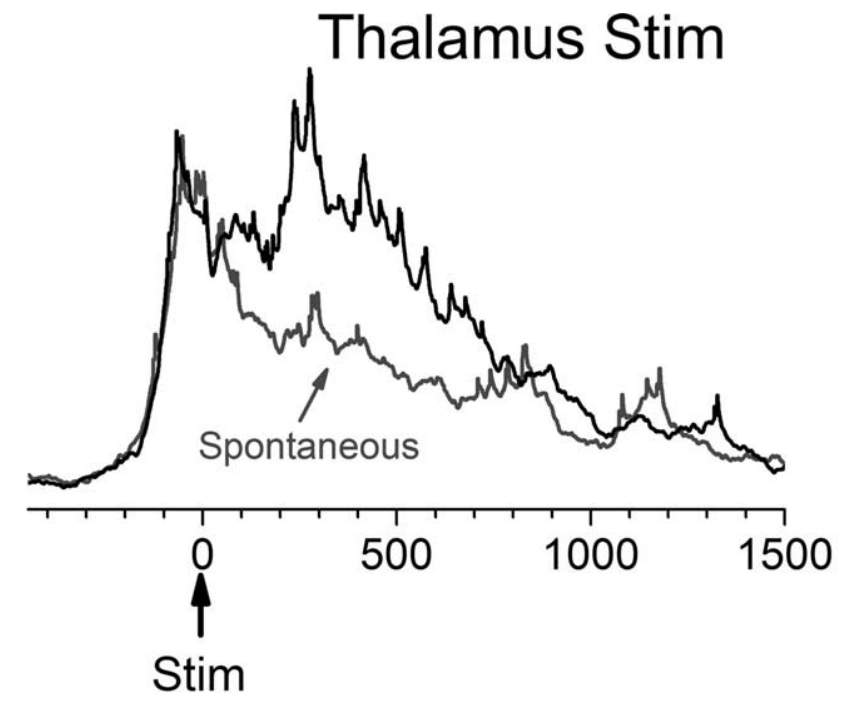

Cortex Stim
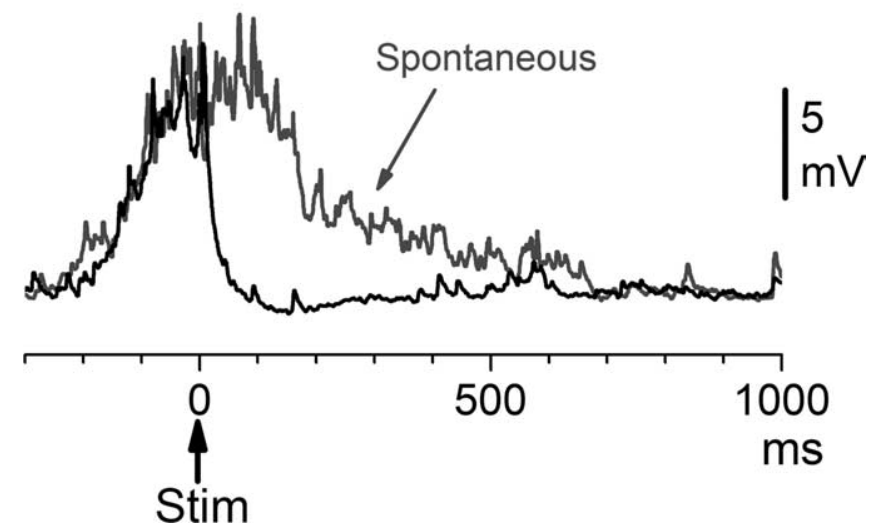

Figure 10. Effects of thalamic or cortical electrical stimulation (Stim) during Up states. Intracellular recordings showing the average depolarization of 30 detected spontaneous Up states (dark gray traces) and the average depolarization of the same number of spontaneous Up states affected by thalamus stimulation (top, black trace) or cortex stimulation (bottom, black trace) delivered during the Up state. The stimulus onset is at zero. Action potentials were removed with a median filter. The bottom and top show different cells.

Up states (MacLean et al., 2005). It is unclear what may be responsible for the differences between our studies, but it may involve the absence of intact corticothalamic connections in their slices, the age of the animals, or other technical differences.

\section{Thalamic activity triggers cortical Up states}

Although it is clear that cortical Up states do not require the thalamus (Steriade et al., 1993a; Sanchez-Vives and McCormick, 2000), cortical and thalamic cells show close phase relations during Up states in vivo (Contreras and Steriade, 1995; Grenier et al., 1998), and thalamic stimulation can trigger strong augmenting responses in cortex (Castro-Alamancos and Connors, 1996a,b). Based on the following results from our study, it is possible that thalamic activity triggers some of the cortical Up states.

First, cross-correlation analysis between VB thalamocortical cells and cortical Up states demonstrates that a significant portion of thalamic spikes in some slices occur immediately before or close to the onset of cortical Up states. This suggests that they could be triggering some of the Up states.

Second, cutting the connections between thalamus and cortex reduces the incidence of cortical Up states. This effect could not be attributed to the cutting procedure or a general change in excitability of the slice but instead seemed to be caused specifically by elimination of thalamocortical connections.

Third, as discussed further below, electrical or chemical stimulation of the thalamus is very effective at triggering and enhancing Up states. Similarly, a previous study found that electrical stimulation of the thalamus at $>10 \mathrm{~Hz}$ triggered cortical Up states in developing mice (MacLean et al., 2005). However, our study further shows that single thalamic stimuli trigger Up states very effectively and enhance spontaneous Up states. We also demonstrate that chemical stimulation of the thalamus triggers cortical Up states, which is important because electrical stimulation may inevitably lead to antidromic stimulation of corticothalamic fibers whose intracortical collaterals recurrently activate cortical cells (Ferster and Lindstrom, 1985; Swadlow, 1989) (for discussion, see Castro-Alamancos, 2004b). Indeed, using histological methods, we found that SI corticothalamic cells in TC slices have intact connections with the VB thalamus, which emphasizes the importance of the chemical thalamic stimulation.

Finally, VB thalamocortical cells can have spontaneous activity in between Up states or even when isolated from the cortex (in non-TC slices), perhaps because thalamocortical cells can produce their own slow oscillation under certain conditions (Hughes et al., 2002). Such spontaneous thalamic activity could be the drive that triggers some of the cortical Up states. In conclusion, these results together indicate that thalamocortical cell activity triggers cortical Up states and enhances spontaneously occurring Up states.

\section{Cortical stimulation mostly suppresses Up states}

Previous work has shown that intracortical electrical stimulation can either start or stop cortical Up states (Shu et al., 2003). Our study agrees and further shows that this depends on the stimulation intensity. Moreover, thalamocortical and intracortical inputs can have opposite effects on Up states. During Down states, cortical stimulation triggers Up states at very low intensities, but at moderate to high intensities, cortical stimulation reduces to nil the probability of evoking an Up state. During Up states, cortical stimulation at moderate to high intensities suppresses the spontaneous Up state. In contrast, thalamic stimulation has somewhat opposite effects. During Down states, thalamic stimulation triggers cortical Up states quite effectively. During Up states, thalamic stimulation enhances the spontaneous Up state. These results indicate that whereas thalamic stimulation has a facilitating effect on Up states, the cortex mostly has a suppressive effect on Up states. Cortical stimuli that stop cortical Up states produce synaptic responses with a more hyperpolarized reversal potential than those produced by cortical stimuli that trigger Up states, indicating stronger activation of inhibition for stopping stimuli (Shu et al., 2003). Thus, the cause for the difference between thalamic and cortical stimulation on Up states may involve a stronger recruitment of inhibition by the cortical stimulation. Indeed, a robust hyperpolarizing potential is triggered by the cortical stimulation with increasing intensities.

\section{Functional relevance}

The high efficacy of thalamic stimulation at evoking cortical Up states across intensities is significant in light of the fact that only a minority of synapses on thalamocortical-recipient cells are thalamic in origin (i.e., $\sim 5-10 \%$ ), and the remaining are intracortical (Benshalom and White, 1986; White, 1989; Douglas et al., 1995). Despite this difference, in the vibrissa system, sensory stimuli drive short-latency cortical responses very effectively dur- 
ing quiescent states, when slow oscillations are present (CastroAlamancos and Oldford, 2002; Castro-Alamancos, 2004a). Moreover, sensory stimuli applied in vivo appear to also drive cortical Up states in quiescent animals (Anderson et al., 2000; Brecht and Sakmann, 2002; Petersen et al., 2003). However, sensory cortical responses are much more suppressed in aroused/ attentive animals (Castro-Alamancos and Oldford, 2002; CastroAlamancos, 2004a). Thus, in dynamic thalamocortical networks in vivo, several interrelated factors will affect cortical responses, such as synchrony among thalamocortical cells (Alonso et al., 1996; Bruno and Sakmann, 2006), the type (burst-tonic) and frequency of preceding thalamocortical cell activity (Swadlow and Gusev, 2001; Castro-Alamancos and Oldford, 2002; Hirata and Castro-Alamancos, 2006), the depression state of thalamocortical synapses (Castro-Alamancos, 2002b, 2004a; CastroAlamancos and Oldford, 2002; Chung et al., 2002; Boudreau and Ferster, 2005), the recruitment of cortical inhibition (Swadlow, 1995; Gibson et al., 1999; Bruno and Simons, 2002; Pinto et al., 2003; Gabernet et al., 2005; Sun et al., 2006), and the modulatory state of the cortex (Gil et al., 1997; Oldford and CastroAlamancos, 2003) and thalamus (Castro-Alamancos, 2002a; Bezdudnaya et al., 2006; Hirata et al., 2006).

An intriguing question is what the functional role of the ability of thalamic activity to trigger Up states and large short-latency sensory responses during quiescent states is. One possibility is that the large sensory cortical responses in quiescent animals serve as a mechanism of heightened sensitivity for detecting transient stimuli (Fanselow and Nicolelis, 1999; Moore et al., 1999; Sherman, 2001; Chung et al., 2002; Castro-Alamancos, 2004a). Indeed, these large evoked responses could serve as a "wake-up call" to alert quiescent animals of the presence of significant sensory stimuli during behavior (Castro-Alamancos, 2004a).

A related possibility is that sustained thalamic activity may have a role in sustaining cortical Up states and converting them into activated cortical states typical of arousal, such as those found after stimulating the brainstem reticular formation (Moruzzi and Magoun, 1949). Apparently, Up states are similar, but not identical, to activated states caused by brainstem reticular formation stimulation (Destexhe et al., 2003; Rudolph et al., 2005) or during natural waking (Steriade et al., 2001); cortical cells have higher input resistance during activated states than during Up states, and synaptic conductance in both of these states is dominated by inhibition. Accordingly, fast-spiking cells (GABA interneurons) discharge robustly during Up states (Hasenstaub et al., 2005). Both fast-spiking cortical cells and thalamocortical cells are strongly driven in vivo by brainstem reticular formation stimulation that produces cortical activation (Castro-Alamancos, 2002b; Castro-Alamancos and Oldford, 2002). Also, waking animals show robust fast-spiking activity (Steriade et al., 1978, 2001; Swadlow, 1995), which would explain the strong inhibitory conductance during Up states and cortical activation. We speculate that a role of sustained thalamocortical cell activity is to keep the sensory cortex in an activated, information-processing state during arousal.

Conversely, another question is the functional role of the suppressive effect of cortical activity on Up states. Such a mechanism has been proposed to underlie the stoppage of persistent activity related to working memory processes (McCormick et al., 2003; Shu et al., 2003). It may also be useful to disengage thalamocortical networks from sensory processing during attentive processing (Castro-Alamancos, 2004b).

\section{References}

Agmon A, Connors BW (1991) Thalamocortical responses of mouse somatosensory (barrel) cortex in vitro. Neuroscience 41:365-379.

Alonso JM, Usrey WM, Reid RC (1996) Precisely correlated firing in cells of the lateral geniculate nucleus. Nature 383:815-819.

Anderson J, Lampl I, Reichova I, Carandini M, Ferster D (2000) Stimulus dependence of two-state fluctuations of membrane potential in cat visual cortex. Nat Neurosci 3:617-621.

Benshalom G, White EL (1986) Quantification of thalamocortical synapses with spiny stellate neurons in layer IV of mouse somatosensory cortex. J Comp Neurol 253:303-314.

Bezdudnaya T, Cano M, Bereshpolova Y, Stoelzel CR, Alonso JM, Swadlow HA (2006) Thalamic burst mode and inattention in the awake LGNd. Neuron 49:421-432.

Boudreau CE, Ferster D (2005) Short-term depression in thalamocortical synapses of cat primary visual cortex. J Neurosci 25:7179-7190.

Brecht M, Sakmann B (2002) Dynamic representation of whisker deflection by synaptic potentials in spiny stellate and pyramidal cells in the barrels and septa of layer 4 rat somatosensory cortex. J Physiol (Lond) 543:49-70.

Bruno RM, Sakmann B (2006) Cortex is driven by weak but synchronously active thalamocortical synapses. Science 312:1622-1627.

Bruno RM, Simons DJ (2002) Feedforward mechanisms of excitatory and inhibitory cortical receptive fields. J Neurosci 22:10966-10975.

Castro-Alamancos MA (2002a) Different temporal processing of sensory inputs in the rat thalamus during quiescent and information processing states in vivo. J Physiol (Lond) 539:567-578.

Castro-Alamancos MA (2002b) Role of thalamocortical sensory suppression during arousal: focusing sensory inputs in neocortex. J Neurosci 22:9651-9655.

Castro-Alamancos MA (2004a) Absence of rapid sensory adaptation in neocortex during information processing states. Neuron 41:455-464.

Castro-Alamancos MA (2004b) Dynamics of sensory thalamocortical synaptic networks during information processing states. Prog Neurobiol 74:213-247.

Castro-Alamancos MA, Calcagnotto ME (1999) Presynaptic long-term potentiation in corticothalamic synapses. J Neurosci 19:9090-9097.

Castro-Alamancos MA, Calcagnotto ME (2001) High-pass filtering of corticothalamic activity by neuromodulators released in the thalamus during arousal: in vitro and in vivo. J Neurophysiol 85:1489-1497.

Castro-Alamancos MA, Connors BW (1996a) Short-term plasticity of a thalamocortical pathway dynamically modulated by behavioral state. Science 272:274-277.

Castro-Alamancos MA, Connors BW (1996b) Cellular mechanisms of the augmenting response: short-term plasticity in a thalamocortical pathway. J Neurosci 16:7742-7756.

Castro-Alamancos MA, Connors BW (1997) Thalamocortical synapses. Prog Neurobiol 51:581-606.

Castro-Alamancos MA, Oldford E (2002) Cortical sensory suppression during arousal is due to the activity-dependent depression of thalamocortical synapses. J Physiol (Lond) 541:319-331.

Castro-Alamancos MA, Rigas P (2002) Synchronized oscillations caused by disinhibition in rodent neocortex are generated by recurrent synaptic activity mediated by AMPA receptors. J Physiol (Lond) 542:567-581.

Castro-Alamancos MA, Rigas P, Tawara-Hirata Y (2006) Resonance $(\sim 10$ $\mathrm{Hz}$ ) of excitatory networks in motor cortex: effects of voltage-dependent ion channel blockers. J Physiol (Lond) 578:173-191.

Chung S, Li X, Nelson SB (2002) Short-term depression at thalamocortical synapses contributes to rapid adaptation of cortical sensory responses in vivo. Neuron 34:437-446.

Contreras D, Steriade M (1995) Cellular basis of EEG slow rhythms: a study of dynamic corticothalamic relationships. J Neurosci 15:604-622.

Cowan RL, Wilson CJ (1994) Spontaneous firing patterns and axonal projections of single corticostriatal neurons in the rat medial agranular cortex. J Neurophysiol 71:17-32.

Cunningham MO, Pervouchine DD, Racca C, Kopell NJ, Davies CH, Jones RS, Traub RD, Whittington MA (2006) Neuronal metabolism governs cortical network response state. Proc Natl Acad Sci USA 103:5597-5601.

Destexhe A, Rudolph M, Pare D (2003) The high-conductance state of neocortical neurons in vivo. Nat Rev Neurosci 4:739-751.

Douglas RJ, Koch C, Mahowald M, Martin KA, Suarez HH (1995) Recurrent excitation in neocortical circuits. Science 269:981-985. 
Fanselow EE, Nicolelis MA (1999) Behavioral modulation of tactile responses in the rat somatosensory system. J Neurosci 19:7603-7616.

Ferster D, Lindstrom S (1985) Synaptic excitation of neurones in area 17 of the cat by intracortical axon collaterals of cortico-geniculate cells. J Physiol (Lond) 367:233-252.

Gabernet L, Jadhav SP, Feldman DE, Carandini M, Scanziani M (2005) Somatosensory integration controlled by dynamic thalamocortical feedforward inhibition. Neuron 48:315-327.

Gibson JR, Beierlein M, Connors BW (1999) Two networks of electrically coupled inhibitory neurons in neocortex. Nature 402:75-79.

Gil Z, Connors BW, Amitai Y (1997) Differential regulation of neocortical synapses by neuromodulators and activity. Neuron 19:679-686.

Grenier F, Timofeev I, Steriade M (1998) Leading role of thalamic over cortical neurons during postinhibitory rebound excitation. Proc Natl Acad Sci USA 95:13929-13934.

Guillery RW (1971) Patterns of synaptic interconnections in the dorsal lateral geniculate nucleus of cat and monkey: a brief review. Vision Res [Suppl] 3:211-227.

Hasenstaub A, Shu Y, Haider B, Kraushaar U, Duque A, McCormick DA (2005) Inhibitory postsynaptic potentials carry synchronized frequency information in active cortical networks. Neuron 47:423-435.

Hirata A, Castro-Alamancos MA (2006) Relief of synaptic depression produces long-term enhancement in thalamocortical networks. J Neurophysiol 95:2479-2491.

Hirata A, Aguilar J, Castro-Alamancos MA (2006) Noradrenergic activation amplifies bottom-up and top-down signal-to-noise ratios in sensory thalamus. J Neurosci 26:4426-4436.

Hughes SW, Cope DW, Blethyn KL, Crunelli V (2002) Cellular mechanisms of the slow $(<1 \mathrm{~Hz})$ oscillation in thalamocortical neurons in vitro. Neuron 33:947-958.

Kao CQ, Coulter DA (1997) Physiology and pharmacology of corticothalamic stimulation-evoked responses in rat somatosensory thalamic neurons in vitro. J Neurophysiol 77:2661-2676.

MacLean JN, Watson BO, Aaron GB, Yuste R (2005) Internal dynamics determine the cortical response to thalamic stimulation. Neuron 48:811-823.

Mao BQ, Hamzei-Sichani F, Aronov D, Froemke RC, Yuste R (2001) Dynamics of spontaneous activity in neocortical slices. Neuron 32:883-898.

McCormick DA, Shu Y, Hasenstaub A, Sanchez-Vives M, Badoual M, Bal T (2003) Persistent cortical activity: mechanisms of generation and effects on neuronal excitability. Cereb Cortex 13:1219-1231.

Metherate R, Ashe JH (1993) Ionic flux contributions to neocortical slow waves and nucleus basalis-mediated activation: whole-cell recordings in vivo. J Neurosci 13:5312-5323.

Moore CI, Nelson SB, Sur M (1999) Dynamics of neuronal processing in rat somatosensory cortex. Trends Neurosci 22:513-520.

Moruzzi G, Magoun HW (1949) Brain stem reticular formation and activation of the EEG. Electroencephalogr Clin Neurophysiol 1:455-473.

Oldford E, Castro-Alamancos MA (2003) Input-specific effects of acetylcholine on sensory and intracortical evoked responses in the "barrel cortex" in vivo. Neuroscience 117:769-778.

Petersen CC, Hahn TT, Mehta M, Grinvald A, Sakmann B (2003) Interac- tion of sensory responses with spontaneous depolarization in layer 2/3 barrel cortex. Proc Natl Acad Sci USA 100:13638-13643.

Pinto DJ, Hartings JA, Brumberg JC, Simons DJ (2003) Cortical damping: analysis of thalamocortical response transformations in rodent barrel cortex. Cereb Cortex 13:33-44.

Rudolph M, Pelletier JG, Pare D, Destexhe A (2005) Characterization of synaptic conductances and integrative properties during electrically induced EEG-activated states in neocortical neurons in vivo. J Neurophysiol 94:2805-2821.

Sanchez-Vives MV, McCormick DA (2000) Cellular and network mechanisms of rhythmic recurrent activity in neocortex. Nat Neurosci 3:1027-1034

Sherman SM (2001) A wake-up call from the thalamus. Nat Neurosci $4: 344-346$.

Sherman SM, Guillery RW (1996) Functional organization of thalamocortical relays. J Neurophysiol 76:1367-1395.

Shu Y, Hasenstaub A, McCormick DA (2003) Turning on and off recurrent balanced cortical activity. Nature 423:288-293.

Steriade M, Oakson G, Kitsikis A (1978) Firing rates and patterns of output and nonoutput cells in cortical areas 5 and 7 of cat during the sleepwaking cycle. Exp Neurol 60:443-468.

Steriade M, Nunez A, Amzica F (1993a) A novel slow $(<1 \mathrm{~Hz})$ oscillation of neocortical neurons in vivo: depolarizing and hyperpolarizing components. J Neurosci 13:3252-3265.

Steriade M, Nunez A, Amzica F (1993b) Intracellular analysis of relations between the slow $(<1 \mathrm{~Hz})$ neocortical oscillation and other sleep rhythms of the electroencephalogram. J Neurosci 13:3266-3283.

Steriade M, Contreras D, Curro DR, Nunez A (1993c) The slow $(<1 \mathrm{~Hz})$ oscillation in reticular thalamic and thalamocortical neurons: scenario of sleep rhythm generation in interacting thalamic and neocortical networks. J Neurosci 13:3284-3299.

Steriade M, McCormick DA, Sejnowski TJ (1993d) Thalamocortical oscillations in the sleeping and aroused brain. Science 262:679-685.

Steriade M, Jones EG, McCormick DA (1997) Thalamus. New York: Elsevier.

Steriade M, Timofeev I, Grenier F (2001) Natural waking and sleep states: a view from inside neocortical neurons. J Neurophysiol 85:1969-1985.

Sun QQ, Huguenard JR, Prince DA (2006) Barrel cortex microcircuits: thalamocortical feedforward inhibition in spiny stellate cells is mediated by a small number of fast-spiking interneurons. J Neurosci 26:1219-1230.

Swadlow HA (1989) Efferent neurons and suspected interneurons in S-1 vibrissa cortex of the awake rabbit: receptive fields and axonal properties. J Neurophysiol 62:288-308.

Swadlow HA (1995) Influence of VPM afferents on putative inhibitory interneurons in S1 of the awake rabbit: evidence from cross-correlation microstimulation, and latencies to peripheral sensory stimulation. J Neurophysiol 73:1584-1599.

Swadlow HA, Gusev AG (2001) The impact of "bursting" thalamic impulses at a neocortical synapse. Nat Neurosci 4:402-408.

White EL (1989) Cortical circuit: synaptic organization of the cerebral cortex-structure, function and theory. Boston: Birkhauser. 